\title{
Do EFL Learners Write with Greater Lexical Complexity: Effects of More Sophisticated Vocabulary Prompt in the Continuation Task
}

\author{
Ziyang Wang ${ }^{1, *}$ Xinan Zhou ${ }^{2}$, Meifang $\mathrm{Li}^{3}$, Dali Hong ${ }^{4}$ \\ ${ }^{1}$ School of international studies, Zhejiang University, Hangzhou, Zhejiang, China \\ ${ }^{2}$ School of International Studies, Zhejiang University, Hangzhou, Zhejiang, China \\ ${ }^{3}$ School of International Studies, Zhejiang University, Hangzhou, Zhejiang, China \\ ${ }^{4}$ School of International Studies, Zhejiang University, Hangzhou, Zhejiang, China \\ *Corresponding author. Email: wzy_arashi@163.com
}

\begin{abstract}
The continuation task, a reading-to-write task that requires students to make the given plot a complete story, is gradually gaining prominence in the National Matriculation English Test in China. Previous empirical studies revealed that alignment effect existed in the continuation task and as students with different proficiency levels actively interact with the source text, their writing performance was facilitated. The present study attempts to investigate whether a more sophisticated vocabulary prompt could facilitate EFL learners' writing performance in the task, specifically from the perspective of lexical complexity. Our findings confirmed the expected alignment effect and discovered that greater lexical complexity resulted from an increase in the concreteness and imageability of the content words, which was viewed as an evidence of a better grasp of the nature of narrative writing required in the continuation task. In addition, the results suggest that the alignment effect is formed due to the activation for more concrete scenes.
\end{abstract}

Keywords: the continuation task, vocabulary prompt, lexical complexity, alignment, EFL.

\section{INTRODUCTION}

More recently the continuation task, a new way of testing a language learner's progress, has gained increasing popularity in China. This manner of testing aims at measuring a learner's reading-to-write skills by requiring them to read and continue an incomplete story. In 2016, this type of task was included in the Zhejiang Province version of National Matriculation English Test (hereafter NMET-ZJ), and will soon be employed in some other provinces such as Jiangsu, Shandong, and Hunan. Preliminary research has confirmed that it assists language teaching and learning and provides positive washback [1]. However, the effects of the taskrelated factors on a test-taker's performance can be explored further. Although some studies have touched upon several task-related factors, such as the test-takers' interest in the source text [2] and the degree of its linguistic complexity [3], the effect of the prompts given has not been systematically examined. Shi, Huang, and $\mathrm{Lu}$ investigated the effect of prompt types in the continuation task and confirmed that more complex prompt types stimulate better writing performance [4]. However, for one specific prompt type, the effect of its level of complexity on the EFL's writing performance remains unclear. Thus, the present study intends to fill this gap in the research by investigating the possible effects of the lexical sophistication of the vocabulary prompt on test-takers' writing performance, with special attention to the lexical complexity. We will first discuss the theoretical basis of the continuation task and empirical researches that support its potential in language learning. We will also discuss its validity and reliability as an instrument for assessing writing. Then, we will review previous researches on lexical sophistication. Research questions will be presented at the end of this section.

\subsection{The Continuation Task}

The continuation task is a special writing task in which test-takers are provided with an incomplete story. 
The ending of the story is removed and test-takers are required to write a coherent and logical continuation of the story upon reading it. This task was at first proposed as an activity with great potential in promoting L2 learning [5]. Such proposition is based upon Pickering and Garrod's Interactive Alignment Model (IAM), which explains the mechanism behind a successful interpersonal interaction [6]. According to this model, there tends to be a coordinated alignment of mental representations on the situational and linguistic level between interlocutors in a dialogue, which is the key to a successful interaction. Based on this theory, Wang and Wang speculated that alignment also exists in the continuation task since the provision of an open-ended source text gives learners a contextual and linguistic basis to complete the writing task and thus promotes learning through facilitating an alignment between the learner and the source text [7]. Empirical studies confirmed that the continuation task can help to inspire imagination, combine comprehension and production, interact with given tests and authors, stimulate creative thinking, promote the ability to self-correct. The task also fits for students with different proficiency levels and makes plagiarism almost impossible [8].

The potential of the continuation task in promoting L2 learning was gradually recognized by researchers. In order to magnify the facilitating role of this task in L2 learning, a multitude of studies have made attempts to investigate the factors that may have influence on the alignment effect from various aspects. This includes how interesting the text is to the reader [2], task instruction [9], linguistic complexity of the source text [3], and so on. However, the prompt effect has not yet been systematically examined. Only Shi et al. investigated the effect of the prompt type in the continuation task. Four groups of Chinese EFL learners participated in a continuation task with four different prompts, namely, bare prompt, framed prompt, vocabulary prompt, and framed vocabulary prompt [4]. Results showed that the more complex prompt type significantly improved the participants' overall writing scores, syntactic complexity, cohesion, as well as source-use features [4]. Thus, their study confirmed that a more complex prompt type stimulates a better writing performance.

However, for one specific prompt type, it is still unknown whether complexity will also influence the writing performance. The latter question might be more practical since the test requirement is fixed in NMET (most tests adopt framed vocabulary prompt, which means test-takers must complete the story based on the opening sentence of each paragraph provided and use at least five keywords underlined in the given passage) and only the inner complexity can be manipulated. Previous research on the continuation task has mainly focused on college-level learners [3][9], while the adoption of this task in many provinces appeals for paying more attention to high school learners and assessment contexts. The current study aims to fill these gaps.

\subsection{Lexical Sophistication of Vocabulary Prompt}

The prompt is used as a critical task-related variable that may have an effect on test-takers' writing performance. Theoretically, it is similar to the studies from the task complexity perspective. There are two competing hypotheses: Cognition Hypothesis and Trade-off Hypothesis, which makes predictions about how varied task complexity impacts a test-taker's linguistic performance.

The prompt given may affect how a test-taker approaches the task and the amount of effort they put into finishing the task. According to Robinson's Cognition Hypothesis, increasing a task's complexity can push learners to use specific language forms and grammaticalize the concepts and functions required for the task [10]. In contrast, Skehan's Trade-off Hypothesis claims that a learner's concentration is limited [11]. The more demanding tasks require learners to invest more attention, which leads to trade-off effects among the three aspects of language production, namely fluency, accuracy, and complexity. As soon as the learners reach their attentional limits, they firstly focus on language meaning (fluency) instead of form (complexity and accuracy). However, contradictions remain about which aspects of language production are being traded off [12].

Besides requiring test-takers to develop a logical and coherent story, the prompt of the continuation task in NMET includes two other elements that would increase a test-taker's workload. Test-takers need to turn their attention to the frame of the story based on the opening sentence of each paragraph on the one hand, and to use five of the ten underlined words in the text on the other hand. Considering the cognition hypothesis discussed above, we aim to gain an understanding of the potential effects of prompts with various levels of complexity on a test-taker's writing performance. To this end, we manipulate the complexity of the vocabulary prompt (i.e., the underlined keywords) by changing their lexical sophistication, which is discussed below in more detail.

The construct of lexical sophistication consists of the depth and the breadth of lexical knowledge available to speakers, readers, and writers [13][14]. In the current study, we use this term to refer to the lexical characteristics of the vocabulary prompt. There are a number of indices to measure the depth and breadth of lexical sophistication in L2 learners that have been proposed, such as using a corpus-derived frequency count [15][16]. or measuring frequency based on word counts taken from a representative corpus [17]. Finally, psycholinguistic properties of words such as 
concreteness, familiarity, imageability, meaningfulness have also been tied to lexical sophistication [17].

\section{THE PRESENT STUDY}

In this study, we examine the effect of lexical sophistication of a vocabulary prompt on the writing performance of Chinese EFL learners at the high school level. They will be completing a continuation task. We seek to address the following research questions:

(1) Do EFL learners write with greater lexical complexity when a more sophisticated vocabulary prompt is presented in the continuation task?

(2) If there exists such an effect, will it be modulated by writing proficiency levels? If so, test takers of which proficiency group will benefit the most from the prompt effect?

\section{METHODOLOGY}

\subsection{Participants}

123 10th-grade EFL learners from two classes at a high school in Jiangsu Province were selected as our participants. The students of one class completed the easier task $(\mathrm{N}=57)$ and those of the other completed the difficult version $(\mathrm{N}=66)$. All students had previously participated in English continuation task before and were thus familiar with the requirements of the task. To confirm the initial comparability of the easy prompt group and difficult prompt group, we obtained their scores on the continuation tasks from their most recent midterm exam. An independent-samples t-test was conducted on the scores using SPSS 25.0 for Windows. Before performing the analysis, Log 10 transformation was used to correct the negative skew in the distributions. No statistically significant difference in the mean writing scores from their midterm was found between the two groups overall $(t=0.68, p=0.50)$.

\subsection{Materials}

The continuation task we chose is a mock test given in the Zhejiang Province. We chose this task to make sure that the participants have not seen it before. In the passage, ten words were underlined in order to direct the test takers' attention to the key elements of the story. Of the ten words, the students are required to use five in their own writing. In order to guide the development of the story, the first sentence of each paragraph is already provided. There were two versions of the task. The vocabulary in the prompts differed in their sophistication but shared similar meanings. Each version was administered to one class of students.

We used the formula offered by $\mathrm{Hu}, \mathrm{Gao}$, and $\mathrm{Lu}$ [18], i.e. " $b=\mathrm{F} \times 0.44+\mathrm{L} \times 0.30+\mathrm{H} \times 0.26$ " to calculate the vocabulary sophistication of two sets of underlined words. Vocabulary sophistication b is described as the weighted sum of $\mathrm{F}$ (frequency), L (length), and $\mathrm{H}$ (coordination between pronunciation and writing). Results from the independent-sample t-test suggested a significant difference between the means of the vocabulary prompt difficulty of the two versions, $\mathrm{t}=$ 2.37, $\mathrm{p}=0.03$. All words appearing in the source text were among the list of words expected to be mastered by 12th-grade learners in China. Three of these words that might have been more challenging were glossed in Chinese.

\subsection{Procedure}

A parcel containing 140 task sheets and 140 answer sheets was sent to the school. Two teachers gave out the exam papers to their own classes and asked their students to finish the task within 30 minutes. Teachers read the task instructions on the task sheet to ensure that all students understood them. When time ran out, answer sheets were handed in and sent back to the researchers. Before successively entering the students' writings into Coh-Metrix 3.0, typos were corrected as misspellings cannot be recognized by the system. The vocabulary prompts used in the continuations were deleted, since they differ in terms of their difficulty and frequency. This could lead to differences in the lexical complexity of students' continuations.

\subsection{Measures}

Since our study's aim is to look into whether the sophistication of the vocabulary prompt has an effect on the lexical complexity of an EFL learner's writing, rather than that of their writing skills in general, holistic scoring was unnecessary despite a total score of 25 was informed beforehand in task instruction. In such a case, we only focus on the measurement of a test takers' lexical complexity in the writing they produced. According to $\mathrm{Lu}$, lexical complexity refers to the variation and sophistication of the words in a text. $\mathrm{He}$ constructed a multidimensional model to divide the concept into lexical density, lexical sophistication, and lexical variation [19]. This can be illustrated as the ratio of the number of content words to the total number of words, the proportion of relatively unusual or advanced words, and the range of a learner's vocabulary as displayed in a text [19]. However, when it comes to the quantification of lexical complexity in the specific writing task, four indices from Coh-Metrix were adopted in a recent study concerning the effect of prompt type, namely MTLD (Measure of Textual Lexical Diversity), Incidence of content words (i.e., the sum of noun incidence, adverb incidence, adjective incidence, and verb incidence per 1,000 words), WRDCacwm (mean concreteness for content words) and WRDlacwm (mean imageability for content words) [4]. 
The measurement of lexical complexity in the present study employed the same features.

\subsection{Data Analysis}

A quantitative analysis was employed to pinpoint the effect of the vocabulary prompt on the lexical complexity of participants' continuations. K-Means clustering was used to select two groups of learners (high-level and intermediate-level) based on their performance in the continuation task that was part of the most recent midterm exam. Independent-samples t-tests were then performed to ensure the comparability of the writing proficiency of the remaining participants in the two prompt groups and to verify if there are any significant differences across the proficiency groups classified by K-Means. Finally, a one-way MANOVA and a set of two-way ANOVAs were conducted to study the main effect of writing proficiency and vocabulary prompt on the four dependent variables, specifically the four features of lexical complexity. The effects of the interaction between the two factors were also examined.

\section{RESULTS}

\subsection{Descriptive Statistics}

Students were divided into three groups based on their performance in the continuation task on the most recent midterm, i.e. high proficiency group $(\mathrm{N}=62)$, intermediate proficiency group $(\mathrm{N}=51)$, and low proficiency group $(\mathrm{N}=10)$. Considering our research question and the relatively small sample size of the low proficiency group, only the data of high proficiency and intermediate proficiency students were kept. Ultimately, we had four groups of participants, namely high proficiency students taking the difficult version $(\mathrm{N}=35$, $\mathrm{M}=16.19, \mathrm{SD}=0.68$ ), high proficiency students taking the easy version $(\mathrm{N}=27, \mathrm{M}=16.39, \quad \mathrm{SD}=0.75)$, intermediate proficiency students taking the difficult version $(\mathrm{N}=29, \mathrm{M}=13.98, \mathrm{SD}=1.17)$, and intermediate proficiency students taking the easy version $(\mathrm{N}=22$, $\mathrm{M}=14.23$, $\mathrm{SD}=0.96$ ). Results from the independentsamples t-tests show no significant differences between groups regarding the writing proficiency of the remaining participants, $\mathrm{t}=0.86, \mathrm{p}=0.39$. Significant differences were found between proficiency groups, $\mathrm{t}=12.41, \mathrm{p}=0.00$.

Table 1 contains the means and the standard deviations of lexical complexity as a function of the two factors, namely prompt and proficiency. Overall, the difficult prompt group received a higher mean score on all four features than the easy prompt group regardless of the participants' writing proficiency level. Also, the participants in the high proficiency group received a higher mean score than the intermediate proficiency group. This goes for all features except for the incidence of content words, regardless of which form of prompt they are provided with.

\subsection{Four Lexical Complexity Features}

Significant differences were found between the two prompts regarding lexical complexity (Wilks's L $=0.908$, $\left.\mathrm{F}(4,106)=2.676, \quad \mathrm{p}=0.036, \quad \eta^{2}=0.092\right)$. The results revealed no significant differences between the proficiency groups (Wilks's L=0.950, $\mathrm{F}(4,106)=1.387$, $\mathrm{p}=0.244, \eta^{2}=0.050$ ) nor for the interaction (Wilks's $\left.\mathrm{L}=0.971, \mathrm{~F}(4,106)=0.798, \mathrm{p}=0.529, \eta^{2}=0.029\right)$. A set of ANOVAs were then conducted to evaluate the effects of the prompts and the writing proficiency on four features of lexical complexity of students' continuations separately. The means and standard deviations for four features of each group are presented in Table 2. The Levene test shows that none of the four features violated the assumption of homogeneity of variance.

Table 3 summarizes the ANOVA results, that is, the main effect of the prompt and proficiency as well as the interaction effect on four lexical complexity features of students' continuations. The ANOVA results indicate no significant interaction between prompt and proficiency for none of the four features. But significant main effects were found for the prompt regarding its concreteness $\left(\mathrm{F}(1,109)=4.554, \mathrm{p}=0.035, \eta^{2}=0.040\right)$ and imageability for content words $(\mathrm{F}(1,109)=7.110$, $\left.\mathrm{p}=0.009, \eta^{2}=0.015\right)$ and for proficiency on MTLD $\left(\mathrm{F}(1,109)=4.803, \mathrm{p}=0.031, \eta^{2}=0.042\right)$. The prompt's main effect suggests that participants from difficult prompt groups tended to show greater lexical complexity in terms of concreteness and imageability for content words than participants from the easy prompt group. Meanwhile, the students' proficiency implies that students with high writing proficiency scored higher on the MTLD feature than the other proficiency group.

\section{DISCUSSION}

\subsection{Prompt effect on the Lexical Complexity}

Our results revealed significant effects of prompt on lexical complexity in the participants' continuations. A more detailed investigation was conducted to explore which lexical features were improved. Results showed that the more sophisticated prompt yielded higher scores on the concreteness and imageability for content words, but not on the lexical diversity and the incidence of content words.

In terms of overall lexical complexity in the testtakers' continuations, the more sophisticated prompt elicited significantly higher mean scores. The results corroborate the previous finding conducted by Shi et al., claiming that writing prompts of higher cognitive demands may lead students to produce higher-quality essays [4]. This finding may be accounted for 
Robinson's Cognition Hypothesis, which posited that increasing a task's complexity can push learners to allocate more attentional resources to the complexity of language, resulting in better lexical complexity [10].

Table 1. Means and Standard Deviations on lexical complexity for the two groups

\begin{tabular}{|c|c|c|c|c|c|c|c|c|c|}
\hline \multirow[t]{2}{*}{ Factor } & & \multicolumn{2}{|c|}{$\begin{array}{c}\text { Measure of lexical } \\
\text { diversity }\end{array}$} & \multicolumn{2}{|c|}{$\begin{array}{c}\text { Incidence of content } \\
\text { words } \\
\end{array}$} & \multicolumn{2}{|c|}{$\begin{array}{c}\text { Concreteness of } \\
\text { content words }\end{array}$} & \multicolumn{2}{|c|}{$\begin{array}{l}\text { Imageability of } \\
\text { content words }\end{array}$} \\
\hline & & $\bar{M}$ & SD & $M$ & SD & $\mathrm{M}$ & SD & $M$ & SD \\
\hline Prompt & Easy & 71.4 & 12.1 & 490.1 & 35.7 & 372.6 & 17.0 & 400.9 & 16.6 \\
\hline Proficiency & $\begin{array}{l}\text { Difficult } \\
\text { High } \\
\text { Intermediate }\end{array}$ & $\begin{array}{l}74.6 \\
75.9 \\
70.0\end{array}$ & $\begin{array}{l}15.7 \\
14.3 \\
13.6\end{array}$ & $\begin{array}{l}495.8 \\
492.3 \\
494.5\end{array}$ & $\begin{array}{l}32.4 \\
36.8 \\
30.2\end{array}$ & $\begin{array}{l}379.1 \\
376.2 \\
376.4\end{array}$ & $\begin{array}{l}17.6 \\
18.0 \\
17.2\end{array}$ & $\begin{array}{l}408.8 \\
405.8 \\
404.9\end{array}$ & $\begin{array}{l}15.9 \\
16.1 \\
17.3\end{array}$ \\
\hline
\end{tabular}

Table 2. Means and Standard Deviations for four lexical complexity features

\begin{tabular}{|c|c|c|c|c|c|c|c|c|c|c|}
\hline \multirow[t]{2}{*}{ Prompt } & \multirow[t]{2}{*}{ Proficiency } & \multirow[t]{2}{*}{$\mathrm{N}$} & \multicolumn{2}{|c|}{$\begin{array}{c}\text { Measure of } \\
\text { lexical diversity }\end{array}$} & \multicolumn{2}{|c|}{$\begin{array}{l}\text { Incidence of } \\
\text { content words }\end{array}$} & \multicolumn{2}{|c|}{$\begin{array}{l}\text { Concreteness of } \\
\text { content words }\end{array}$} & \multicolumn{2}{|c|}{$\begin{array}{l}\text { Imageability of } \\
\text { content words }\end{array}$} \\
\hline & & & $M$ & SD & $M$ & SD & $M$ & SD & $M$ & SD \\
\hline \multirow[t]{2}{*}{ Easy } & High & 27 & 74.2 & 11.8 & 491.7 & 41.7 & 375.1 & 18.2 & 403.3 & 16.5 \\
\hline & Intermediate & 22 & 68.0 & 11.9 & 488.2 & 27.4 & 369.4 & 15.2 & 398.0 & 16.6 \\
\hline \multirow[t]{2}{*}{ Difficult } & High & 35 & 77.2 & 16.1 & 492.9 & 33.1 & 377.0 & 18.1 & 407.6 & 15.8 \\
\hline & Intermediate & 29 & 71.6 & 14.9 & 499.4 & 31.8 & 381.7 & 17.0 & 410.2 & 16.1 \\
\hline
\end{tabular}

Table 3. Results of the two-way ANOVA for four lexical complexity features

\begin{tabular}{|c|c|c|c|c|c|c|c|c|c|c|c|c|}
\hline \multirow[t]{2}{*}{ Factor } & \multicolumn{3}{|c|}{$\begin{array}{l}\text { Measure of lexical } \\
\text { diversity }\end{array}$} & \multicolumn{3}{|c|}{$\begin{array}{c}\text { Incidence of content } \\
\text { words }\end{array}$} & \multicolumn{3}{|c|}{$\begin{array}{l}\text { Concreteness of } \\
\text { content words }\end{array}$} & \multicolumn{3}{|c|}{$\begin{array}{l}\text { Imageability of } \\
\text { content words }\end{array}$} \\
\hline & $\mathrm{F}$ & Sig & $\eta^{2}$ & $\mathrm{~F}$ & Sig & $\eta^{2}$ & $\mathrm{~F}$ & Sig & $\eta^{2}$ & $\mathrm{~F}$ & Sig & $\eta^{2}$ \\
\hline$\overline{\text { Prompt }}$ & 1.51 & 0.22 & 0.01 & 0.91 & 0.34 & 0.01 & 4.55 & $0.04^{*}$ & 0.04 & 7.11 & $0.01^{*}$ & 0.06 \\
\hline Proficiency & 4.80 & $0.03^{*}$ & 0.04 & 0.05 & 0.82 & 0.00 & 0.02 & 0.89 & 0.00 & 0.22 & 0.64 & 0.00 \\
\hline Prompt ${ }^{*}$ Proficiency & 0.01 & 0.91 & 0.00 & 0.60 & 0.44 & 0.01 & 2.50 & 0.12 & 0.02 & 1.63 & 0.21 & 0.02 \\
\hline
\end{tabular}

Note. $* \mathrm{p}<0.05$

However, for the specific lexical features, it is somewhat surprising that only the concreteness and imageability for content words were improved, but not the lexical diversity or the incidence of content words, as the provision of the more sophisticated prompt was at first expected to affect test-takers' lexical choice generally. A possible explanation may lie in the nature of the difficult prompt. This study adapted the same writing task into two versions with their vocabulary prompts differing in terms of lexical sophistication but sharing similar meaning, for example, "disrespectful behaviors", "virtuous things" and "pupils" in the difficult version compared with "bad behaviors, "good things" and "students" in the simple version. If analyzed thoroughly, the more sophisticated prompt itself is more concrete and imaginable since it narrows down possible conditions. It is assumed that concrete and imaginable prompts will stimulate test-takers' imagination of specific scenes and expressions, thus making their continuations more vivid. For example, the sophisticated prompt "disrespectful behaviors" may render test-takers think of scenes like "have a fight" or "speaking rudely" where respect is not shown, while the simple prompt "bad behaviors" just activates vague images where unpleasant results are triggered. All in all, the provision of the more sophisticated prompt may have cued the test-takers to use more words and phrases with higher concreteness and imageability, leading to the expected emergence of alignment.

As to the lexical diversity and the incidence of content words, the results differ from our prediction that the more difficult vocabulary prompt would elicit more fluent and complex writing samples than the simple prompt. These results are consistent with Shi, Huang, and Lu's finding that none of the fluency, grammatical accuracy, and lexical complexity showed significant differences irrespective of prompt types [4]. This result may have arisen from the proficiency level of the participants in the two studies. Participants in both studies were EFL learners at high schools and their language proficiency was not high enough so that their vocabulary size was not sparsely divided to show a great difference. Although the more sophisticated prompt encouraged them to use more complex expressions, they would probably fail to exhibit the alignment effect due to their limited vocabulary size. This is indicated by the result that the difficult prompt group got higher scores on lexical diversity and the incidence of content words than the easy prompt group, though not significantly different. What's more, the grouping method in this study needs improvement. We divided students into different proficiency groups based on the scores they earned in the midterm. However, the score cannot fully 
represent the students' vocabulary size, since the scores are not only judged based on vocabulary. Students who got a high score may have a good command of grammar. Thus, the comprehensive understanding of the effect of prompt sophistication on test-takers' lexical complexity warrants further research which accurately divides students based on their vocabulary size, for example, by standard vocabulary test.

\subsection{Modulating Effect of Writing Proficiency on the Vocabulary Prompt}

The two-way ANOVA results showed no significant main effect of the writing proficiency on test-takers' lexical complexity, except on lexical diversity. What's more, the results indicated no significant interaction between prompt and proficiency for neither of the four features. These results indicate the alignment effect of vocabulary prompt sophistication on lexical complexity in L2 learners' writing is not influenced by proficiency levels, which partially corroborates previous findings that the continuation task is fit for students with different proficiency levels [8]. However, this conclusion should be interpreted with caution. It remains to be seen whether the test-takers' vocabulary size influences the lexical complexity of their continuations.

\section{CONCLUSION}

The continuation task is gaining popularity in highstakes testing contexts. The purpose of the present study is to investigate whether a more sophisticated vocabulary prompt could facilitate EFL learners' performance in the task, specifically from the perspective of lexical complexity. Our findings revealed that the expected alignment effect was found and thus confirmed our supposition that EFL learners were able to present a higher level of lexical complexity when more difficult words within their understanding were encountered. With further analysis, we discovered that behind such facilitation was an increase in the concreteness and imageability of the content words, which was viewed as evidence of a better grasp of the nature of narrative writing required in the continuation task. To be more specific, our findings suggest that the alignment effect is formed due to the activation for more concrete scenes on the grounds that the words with greater sophistication were assumed to have higher constraints on their meaning. As a result, during the language teaching and learning process, especially in the training of completing the continuation task, a moderately more difficult vocabulary prompt may enable learners to demonstrate their writing ability more fully.

There are still some limitations in the current study. First of all, the criterion for underlining words in the original task is not clearly defined. Apart from merely adjusting the sophistication of the words, more attention can be attached to what words are underlined and the reasons why they are chosen in further research. Also, Students' latest score on the continuation task was used to represent their writing proficiency and they were accordingly divided into three groups of different levels. It is worth mentioning that although they were deemed EFL learners with various levels of writing ability when compared with each other, their proficiency might not be discrepant enough to exhibit a practically significant difference in the alignment effect in the task. Moreover, we encourage future studies to include an overall rating of the writings. With scores graded by experienced raters, questions such as whether higher lexical complexity indicates higher scores, whether prompt effect exists in the results of grading and whether such effect is influenced by proficiency can be further explored.

\section{ACKNOWLEDGMENTS}

This paper has been supported by the College Students' Science and Technology Innovation Project and University Youth Talents Supporting Program of Zhejiang Province, China (Project No. 2021R401223).

\section{REFERENCES}

[1] Wang, C. (2015). Why does the continuation task facilitate L2 learning? Foreign Language Teaching and Research, 47(5), 753-762.

[2] Guo, Y., Wang, X. (2019). Effect of reading materials on writings in the continuation task. Journal of Donghua University(Social Science), 19(04), 425-435.

[3] Peng, J., Wang, C., \& Lu, X. (2020). Effect of the linguistic complexity of the input text on alignment, writing fluency, and writing accuracy in the continuation task. Language Teaching Research, 24(3). https://doi.org/10.1177/1362168818783341

[4] Shi, B., Huang, L., \& Lu, X. (2020). Effect of prompt type on test-takers' writing performance and writing strategy use in the continuation task. Language Testing, 37(2), 361388. https://doi.org/10.1177/0265532220911626

[5] Wang, C. (2016). The role of the continuation task in NMET in promoting English learning. English Learning, 11, 10-11.

[6] Pickering, M., \& Garrod, S. (2004). Toward a mechanistic psychology of dialogue. Behavioral \& Brain Sciences, 27(2), 169-190. https://doi.org/10.1017/s0140525x04000056 
[7] Wang, C., \& Wang, M. (2015). Effect of alignment on L2 written production. Applied Linguistics, $36(5)$, 503-526. https://doi.org/10.1093/applin/amt051

[8] Wang, C. (2012). The continuation task: an effective method in enhancing foreign language acquisition. Foreign Language World, 5, 2-7.

[9] Yuan, D. (2013). The effect of task instructions on L2 writing from the perspective of alignment (Unpublished master's thesis). Guangdong University of Foreign Studies, Guangzhou, China.

[10] Robinson, P. (2001). Task complexity, task difficulty, and task production: Exploring interactions in a componential framework. Applied Linguistics, 22(1), 27-57. https://doi.org/10.1093/applin/22.1.27

[11] Skehan, P. (1998). A cognitive approach to language learning. Oxford University Press. https:// doi.org/10.2307/3587891

[12] Yuan, F., \& Ellis, R. (2003). The effects of pre-task planning and on-line planning on fluency, complexity and accuracy in L2 monologic oral production. Applied Linguistics, 24(1), 1-27.

[13] Meara, P. (1996). The dimensions of lexical competence. In G. Brown, K. Malmkjaer \& J. Williams (Eds.), Performance and competence in second language acquisition (pp. 35-53). Cambridge, England: Cambridge University Press.

[14] Read, J. (1998). Validating a test to measure depth of vocabulary knowledge. In A. Kunnan (Ed.), Validation in language assessment (pp. 41-60). Mahwah, NJ: Lawrence Erlbaum.

[15] Laufer, B. (1994). The lexical profile of second language writing: Does it changeover time? RELC Journal, 25(2), 21-33. doi:10.1177/003368829402500202

[16] Laufer, B., \& Nation, P. (1995). Vocabulary size and use: Lexical richness in L2 written production. Applied Linguistics, 16(3), 307 322. https://doi.org/10.1093/applin/16.3.307

[17] McNamara, D. S., Crossley, S. A., \& McCarthy, P. M. (2010). Linguistic features of writing quality. Written Communication, 27, 57-86. doi:10.1177/074108830935154

[18] Hu, Y., Gao, J., \& Lu, H. (2016). Assessing Lexical difficulty in English Vocabulary Adaptive Testing System. Modern Education Technology, 26(3), 100-106.

[19] Lu, X. (2012). The relationship of lexical richness to the quality of ESL learners' oral narratives. The
Modern Language Journal (Boulder, Colo.), 96(2), 190-208. https://doi.org/10.1111/j.1540-

4781.2011.01232.x 